\title{
DXS6673E Encodes a Predominantly Nuclear Protein, and Its Mouse Ortholog DXHXS6673E Is Alternatively Spliced in a Developmental- and Tissue-Specific Manner
}

\author{
Maurice P. Scheer,* S. van der Maarel,† S. Kübart,* A. Schulz,* J. Wirth,* S. Schweiger,* \\ H.-H. Ropers,* $\neq$ and H. G. Nothwang*,1
}

*M ax Planck Institut für M olekulare Genetik, Innestrasse 73, D-14195 Berlin-Dahlem, Germany; †Department of Human Genetics, University of Leiden, Leiden, The Netherlands; and ¥Department of Human Genetics, University Hospital, Nijmegen, The Netherlands

Received June 28, 1999; accepted October 14, 1999

DXS6673E is a candidate gene for nonspecific $X$-linked mental retardation and encodes a novel Zn-finger protein. The ortholog murine gene DXHXS6673E in XC-D was isolated and characterized. It is ubiquitously expressed in all embryonic stages and adult tissues. Two different transcription start sites exist that result in two major transcripts of 6055 and 5352 nucleotides, each composed of 25 exons. Exon 1A is tissue specific, whereas exon $1 B$ is transcribed constitutively. Both variants are translated into the same 1370-amino-acid protein. Transcripts are subject to alternative splicing at the $5^{\prime}$-end. Some of the isoforms are developmental stage and tissue specific. Among them, one was present only in embryos and adult brain. Sequence analysis demonstrated evolutionary conservation down to the arthropods and defined several conserved protein motifs. Subcellular localization studies with green fluorescent protein as a reporter showed that DXS6673E is predominantly located in the nucleus due to several functional nuclear localization signals. Three distinct protein distribution patterns in COS-7 cells could be identified. $\odot 2000$ Academic Press

\section{INTRODUCTION}

Mental retardation (MR) is a frequent and very heterogeneous disorder that affects about 1-3\% of individuals. Population studies indicate that X-linked genes account for approximately $20-50 \%$ of all cases (XMR). Recently, several genes for nonsyndromic XMR (NSXMR), i.e., MR without additional clinical features, have been isolated, FMR2, oligophrenin-1, GDI $\alpha$ and PAK3, where mutations have been found in independent patients (Gecz et al., 1996; Billuart et al., 1998;

Sequence data from this article have been deposited with the EMBL/GenBank Data Libraries under Accession No. AF 156605.

${ }^{1}$ To whom correspondence should be addressed. Telephone: +49 (30) 8413 1250. Fax: +49 (30) 8413 13831. E-mail: nothwang@ molgen.mpg.de.
d'Adamo et al., 1998; Allen et al., 1998). Another candidate gene, DXS6673E in Xq13, was found to be disrupted by a balanced X;13 translocation in a mentally retarded female (van der Maarel et al., 1996). This gene is composed of 26 exons with two different untranslated exons $1 \mathrm{~A}$ and $1 \mathrm{~B}$. The X-chromosomal breakpoint is located in exon $1 \mathrm{~A}$, and sequences downstream of the breakpoint are still expressed from the derivative chromosome $X$. The gene is highly conserved among vertebrates and most abundantly expressed in brain. It encodes a 1358-amino-acid protein of unknown function. Sequence analysis revealed that it represents the first member of a new gene family, defined by a five-times repeated, novel, conserved $\mathrm{Zn}$ finger-related motif of the general form $\mathrm{CX}_{2} \mathrm{CX}_{19-}$ ${ }_{22} \mathrm{CX}_{3} \mathrm{CX}_{13-19} \mathrm{CX}_{2} \mathrm{CX}_{19-25} \mathrm{FCX}_{3} \mathrm{CX}_{3} \mathrm{~F} / \mathrm{Y}$. It is unknown whether it mediates protein-DNA or protein-protein interactions (Xiao et al., 1998; Smedley et al., 1998; Reiter et al., 1998).

Interestingly, the second member, ZNF 198, was isolated by positional cloning in the study of another genetic disorder, the $t(8 ; 13)$ myeloproliferative syndrome (Xiao et al., 1998; Smedley et al., 1998; Popovici et al., 1998). The reciprocal translocation fuses ZNF 198 to the fibroblast growth factor receptor-1 and thereby causes Iymphoblastic lymphoma. The disorder generally progresses to full-blown acute myelogeneous leukemia within a year of diagnosis. Another member, KI AA0425, was identified by a database search (Reiter et al., 1998).

To gain further insight into the function of this gene family, we isolated and characterized DXHXS6673E (approved MGD nomenclature), the murine ortholog of DXS6673E. This allowed expression studies in various developmental stages and adult tissues. Evolutionary conservation was assessed by searching publicly available databases. To test putative nuclear addresses, subcellular localization studies of DXS6673E-green fluorescent protein (GFP) constructs were performed. 


\section{MATERIALS AND METHODS}

Library screening and sequence analysis. A Lambda ZAP II mouse brain cDNA library (Stratagene) was screened, and Bluescript $\mathrm{KS}(+)$ plasmids were isolated by in vivo excision according to the manufacturer's instructions. To obtain genomic clones, the P1 library 703 (German Resource Centre) containing genomic mouse DNA inserts was screened. Doublestranded sequencing of cDNA clones was performed with labeled universal M13 primers and the Thermo sequenase fluorescence-labeled primer cycle sequencing kit (ABI) on a Licor sequencer (MWG-Biotech). To generate the genomic sequence at the exon-intron boundaries, P1 clones were digested with HindlII and subsequently shotgun-cloned into pBluescript. Selected subclones were sequenced either with M13 or with labeled exon-specific oligonucleotides. Some of the boundaries were characterized by genomic long-range PCR. Sequence analysis was performed with the GCG package (Devereux et al., 1984).

RNA isolation and RT-PCR analysis. Total mouse embryo RNA was isolated by the guanidinium-thiocyanate method (Chomczynski and Sacchi, 1987). Day 1 was defined as day of vaginal plug, and embryos were prepared at 9, 12, 14, 16, and 18 days of gestation. RNA from tissues was isolated using the RNAeasy kit (Qiagen), and poly $(A)^{+}$RNA was obtained by subsequent purification on magnetic beads using a mRNA isolation kit (Boehringer Mannheim). For synthesis of CDNA, approximately 40-200 ng of purified mRNA or 100-500 ng of total RNA was randomly primed by using SuperScript II RNase H- reverse transcriptase (Gibco BRL). Reverse transcription was carried out for $40 \mathrm{~min}$ at $42^{\circ} \mathrm{C}, 10 \mathrm{~min}$ at $45^{\circ} \mathrm{C}$, and $10 \mathrm{~min}$ at $50^{\circ} \mathrm{C}$. Negative controls without reverse transcriptase were run under identical conditions. PCR amplification with DXHXS6673E gene-specific primers was performed on $1 / 50$ of the original RT reaction.

Nested PCR was carried out $\left(96^{\circ} \mathrm{C}\right.$ for $30 \mathrm{~s}, 60^{\circ} \mathrm{C}$ for $30 \mathrm{~s}$, and $72^{\circ} \mathrm{C}$ for $2 \mathrm{~min}$ ) in a final volume of $50 \mu \mathrm{l}$ containing $40 \mathrm{pmol}$ of each primer and $1 \mathrm{U}$ of Taq polymerase. Nested primer pairs were as follows: first PCR, forward primer, TTGAATGAGAGGAGGGGATC, and reverse primer, GCTGGTCTTGCCATTACGAT; nested PCR, CTTGAGCCAGGTAAGGAAAG and ACACTGGTCACAACAGTTGG (exon 1A to exon 7); first PCR, CCG TTG CTG TCT GCA GTT CT and GCTGGTCTTGCCATTACGAT; nested PCR, CTT GTA GCGGTT ACT CTA AC and ACACTGGTCACAACAGTTGG (exon 1B to exon 7); first PCR, ACTCCAAGAAGCCCTTGGGC and CACGCTGGCAGGTTTGGGA; nested PCR, GAA AGA CCT GTA CCT TCT GC and TTG CTT GTA CAG CAG TAC AC (exon 5 to exon 12); first PCR, CTGCCGGCAGGAAAAGCTCC and AAT CAG CCT CCA AGT CTT GC; nested PCR, CAC GAG AAG CTT CGA TTC AG and CAT CTA AGA CAT TAG CCA TC (exon 11 to exon 18); and first PCR, GCA GAG GGA CTC CTG GAA GA and CAATGCAGGAGCGCTCAGG; nested PCR, GGA CAG CTC GGG ATG ATG TC and AAC ACA TCA TTT CGA GTT CG (exon 18 to exon 25). One-fifth of the PCR product was analyzed on $1.5 \%$ agarose gels with $0.5 \mu \mathrm{g} / \mathrm{ml}$ ethidium bromide in $1 \times$ TAE

Northern bl ot analysis. A Northern blot of RNA from embryos of stage $9,12,14,16$, and 18 days of gestation was prepared according to standard protocols (Sambrook et al., 1989), and an adult multiple tissue Northern blot was purchased from Clontech. Both blots were hybridized in express hybridization solution (Clontech) with a ${ }^{32} \mathrm{P}$ labeled cDNA insert for $90 \mathrm{~min}$ at $65^{\circ} \mathrm{C}$. Hybridized filters were washed for $2 \times 30 \mathrm{~min}$ in $2 \times$ SSC, $0.1 \%$ SDS, and $30 \mathrm{~min}$ in $0.1 \times$ SSC, $0.1 \%$ SDS at $65^{\circ} \mathrm{C}$. Autoradiography took $16-40 \mathrm{~h}$ at $-70^{\circ} \mathrm{C}$ using two intensifying screens.

GFP constructs and sitedirected mutagenesis. The ORF of DXS6673E was amplified in two overlapping fragments from cDNA clones 50:17 and 50:33 (van der Maarel et al., 1996) by using the proofreading Pfu polymerase (Stratagene). To analyze both $\mathrm{N}$ - and C-terminal fusion of DXS6673E to GFP, fragments were ligated into the green fluorescent protein vectors peGFP-N3 and peGFP-C1 (Clontech) using primers with the respective restriction site for amplification. Site-directed mutagenesis was performed using the
QuickChange kit (Stratagene). Individual clones were sequenced to verify their insert, and Western blot analyses were used to assess full-length translation of fusion constructs. DNA from two independent clones for each construct was purified using the Qiagen plasmid purification kit (Qiagen). To construct C-terminal truncated DXS6673E fusion proteins, fragments were amplified using primers with additional restriction enzyme sites. Constructs $\Delta 655-1358$ and $\Delta 474-1358$ contain amino acid residues (aa) 1- 654 and 1-473, re spectively.

Cell culture and transfection experiments. COS-7 cells were maintained at $37^{\circ} \mathrm{C}$ and $5 \% \mathrm{CO}_{2}$ in Dulbecco's modified Eagle's medium. For fluorescence microscopy analysis, cells were grown directly on untreated glass coverslips. In a typical transfection experiment, $3 \mu \mathrm{g}$ of plasmid DNA was delivered into COS-7 cells using $3 \mu \mathrm{l}$ of Lipofectace transfection reagent (Gibco BRL). Cells were grown to about $60 \%$ confluence, exposed to lipid-DNA complexes for $5 \mathrm{~h}$, and grown for an additional 24 to $72 \mathrm{~h}$ until analyzed. Two independent clones per construct were used.

Fluoresence microscopy. Expression of GFP was analyzed either immediately after incubation in living cells or in formaldehyde-fixed cells. For formaldehyde fixation, cells were washed once in phosphate-buffered saline (PBS), once in PBS with $2 \%$ paraformaldehyde, and three times again in PBS, $0.1 \%$ Triton, dried, and mounted onto a microscope slide in DAPI (4,6-diamidino-2-phenylindol dilactate; Boehringer Mannheim) antifade mounting solution $(0.4 \mu \mathrm{g} / \mathrm{ml}$ DAPI in a solution consisting of 9 parts of glycerol containing $2 \%$ 1,4-diazobicyclo-(2-2-2)-octane and one part $0.2 \mathrm{M}$ Tris- $\mathrm{HCl}, \mathrm{pH} 7.5$ ). Alternatively, the cells were briefly washed in PBS and mounted onto a microscope slide in PBS. To prevent cells from drying, the coverslips were sealed to the microscopic slides with rubber cement. Green fluorescence staining and DAPI nuclear staining were analyzed with an epifluorescence microscope (Axioskop 50; Zeiss). Photos were taken with a CCD camera. For each clone, 200-300 cells were visually scored.

Subcelular fractionation and Western bl ot analysis. After transfection, cells were transferred to $150-\mathrm{ml}$ plastic bottles and grown for 24-72 h. Thereafter, cells were washed with ice-cold PBS, scraped from the dishes and pelleted. The pelleted cells were washed twice with isotonic buffer (40 mM Hepes, $\mathrm{pH} 7.4,0.32 \mathrm{M}$ sucrose, $1 \%(\mathrm{v} / \mathrm{v})$ $\beta$-mercaptoethanol, and protease inhibitors (Boehringer Mannheim) and then resuspended in the same buffer. The cells were disrupted by dounce homogenization (10 strokes) in $2 \mathrm{ml}$ of the buffer. The nuclei were separated from the cytoplasm by low-speed centrifugation (1000g for $10 \mathrm{~min}$ ), and both fractions were collected. Nuclei were then resuspended in $2 \mathrm{ml}$.

For Western blot analysis, $150 \mu$ l of the nuclear fraction and $150 \mu \mathrm{l}$ of the cytoplasmic fraction were acetone-precipitated and separated on an 8\% SDS-polyacrylamide gel (Bio-Rad) and el ectroblotted (BioRad) onto Hybond-N nitrocellulose membranes (Amersham Pharmacia). After being blocked with $5 \%$ defatted dried milk solution, the filters were incubated with a 1:1000 dilution of polyclonal antisera against GFP (Clontech) for $1 \mathrm{~h}$ at room temperature. Subsequently, the blots were incubated with horseradish peroxidase-conjugated anti-rabbit Fab fragments (Amersham Pharmacia) at a 1:2000 dilution and developed with enhanced chemiluminescence reagents (Amersham Pharmacia) as specified by the manufacturer.

\section{RESULTS}

\section{Isolation of DXHXS6673E}

To isolate the murine ortholog of DXS6673E, a mouse fetal brain cDNA library was screened with several human subclones. Seventeen cDNA dones that showed significant sequence similarity were isolated. The composite cDNA corresponds to exons $1 \mathrm{~b}-25$ of the human gene (data not shown). No clone similar to the human alternative first exon $1 \mathrm{~A}$ could be isolated by 
library screening or by RT-PCR using primers derived from the human sequence. Partial sequencing of $\mathrm{P} 1$ clone ICRFP703J 20233, which contained a large part of DXHXS6673E (see below), however, resulted in a sequence similar to human exon $1 \mathrm{~A}$, and by RT-PCR, a 703-bp product could be amplified from various mouse tissues (see below). To check whether exon $1 \mathrm{~A}$ and $1 B$ represent two alternative transcription initiation sites, RNA populations from various developmental stages $(12,14,16$, and 18 days of gestation) and adult tissues (brain, kidney, liver, placenta, testis, and lung) were screened by nested RT-PCR for the presence of a transcript containing exons $1 A$ and $1 B$. No product was obtained using forward primers in exon $1 A$ and reverse primers in exon 1B. Thus, two alternative untranslated first exons exist in mouse that give rise to two different transcripts of 6055 (variant 1A) and 5352 (variant 1B) nucleotides. Both transcripts encode a protein of 1370 amino acid residues (Fig. 1A).

To study the genomic organization of DXHXS6673E, RT-PCR products were hybridized to a P1 mouse genomic library. Two positive clones were identified: ICRF 703I 2291 and ICRF 703J 20233. Sequence information derived from these clones together with longrange $P C R$ allowed the determination of all exon-intron boundaries. DXHXS6673E consists of 26 exons with the initiation codon AUG in exon 2 as its human counterpart. All splice donor and splice acceptor sites conform to the AG/GT rule. Most exons are in the range between 50 and 300 nucleotides. Exceptions are exons $1 A, 2$, and 25 with 703, 680, and 1419 nucleotides. Cytogenetic analysis of PAC clone ICRF 703J 20233 mapped the gene to chromosome XC-D (data not shown). This assignment corresponds well with the localization of human DXS6673E in Xq13.

\section{Sequence Analysis}

A detailed comparative sequence analysis at both the nucleotide and the amino acid levels between DXS6673E and its murine ortholog revealed sequence identities higher than $90 \%$. Interestingly, both genes contain a dinucleotide repeat in exon $1 A$, but it is composed of (GA) in human and (GT) in mouse (data not shown). At the amino acid level, only two significant differences were observed at positions 238-239 and 797-807, where the published human protein lacks 2 (VQ) and 11 (RTPDENGNLGK) amino acid residues, respectively. RT-PCR experiments and a database search, however, showed that these differences are due to alternative splicing (see below).

Analysis of the amino acid composition revealed that the DXHXS6673E protein contains the Zn-finger motifs characteristic for members of this novel family, too (aa 313-387, 411-486, 500-576, 593-662, 681-744) (Smedley et al., 1998; Reiter et al., 1998) (Fig. 1A). In addition, five nuclear localization signals (NLSs) of the SV40 large $T$ antigen type at positions 234-241 (PPERKRSE), 283-289 (PFRPRRS), 827- 833 (PRKN-
KAA), 1261-1267 (PVRQRKG), and 1272-1278 (PGKRKRE), one bipartite NLS between amino acid residues 479 and 493 (KRFCNTTCLGAYKKKNT), and three putative tyrosine phosphorylation sites at positions 543-551 (RSLSDPCYY), 1130-1138 (RYEPDSIYY), and 1312-1319 (RTRNDVFY) are conserved between mouse and human (Fig. 1A). The phosphorylation sites are close to some NLSs and may modulate the nuclear import as described for several proteins (Dingwall and Laskey, 1998). Finally, there is a proline-rich sequence at position 814-828 that contains two putative SH3-binding motifs PxxP (PSVPTPPPPPPPATP) (Fig. 1A).

A search for further family members yielded, in addition to the known members ZNF 198 and KIAA0425, the human gene ZNF 258, which displays sequence similarity over a long range (39\% identity) (Fig. 1A). However, the encoded protein lacks some cysteines in the C-terminal part of the second, and the N-terminal part of the third, Zn-finger motif (Fig. 1A). This defines the novel $\mathrm{Zn}$-fingers as alternating motifs of types $\mathrm{CX}_{2} \mathrm{CX}_{19-22} \mathrm{CX}_{3} \mathrm{C}$ and $\mathrm{CX}_{2} \mathrm{CX}_{19-25} \mathrm{FCX}_{3} \mathrm{CX}_{3} \mathrm{~F} / \mathrm{Y}$. Sequence similarity in the entire gene family is highest at the C-terminal part with several conserved amino acid sequences, such as two regions with interspersed prolines (PI/VPVPINF/yV/iPV/iP (aa 869-879) and PVPF/ tXXPV/IPV/mPVPM/vF/IL/iP (aa 888-903)).

Several ESTs from rat (GenBank Accession Nos. Al 111670, Al 412415, Al 409797, Al 112706, Al 502308), rabbit (C83185, C83052), turkey (AF 062408), zebrafish (Al496701, Al397030), Al397051), and fruitfly (AA951966, AA567252, AA543240, AA949953) shared significant sequence similarity with DXHXS6673E (Blastp $\mathrm{E}$ values between $3 \times 10^{-39}$ and $4 \times 10^{-6}$; data not shown) and likely represent homologous genes in the respective species. Although these ESTs provided limited sequence information, a detailed analysis revealed the conservation of the putative tyrosine phosphorylation site at position 1312-1319 down to the arthropod Drosophila melanogaster, indicating its functional importance (Fig. 1B). No similarity was found to the almost completely sequenced genome of the nematode Caenorhabditis elegans.

\section{Expression Analysis}

To study the expression of DXHXS6673E, Northern bl ot analyses were performed. The gene is expressed in all developmental stages examined, i.e., 9, 12, 14 16, and 18 days of gestation (Fig. 2). It is also ubiquitously, but to a variable degree, expressed in all adult tissues analyzed. Strongest expression was observed in adult brain and testis (Fig. 2). One major transcript of about 5600 nucleotides was detected. This transcript size corresponds well with the length of variant 1B (5352 nt), taking into account a poly(A)+ tail of about $200-$ $250 \mathrm{nt}$. Some weaker signals were also obtained in the lower and higher molecular weight ranges. To analyze whether some of these fainter signals represented al- 
A

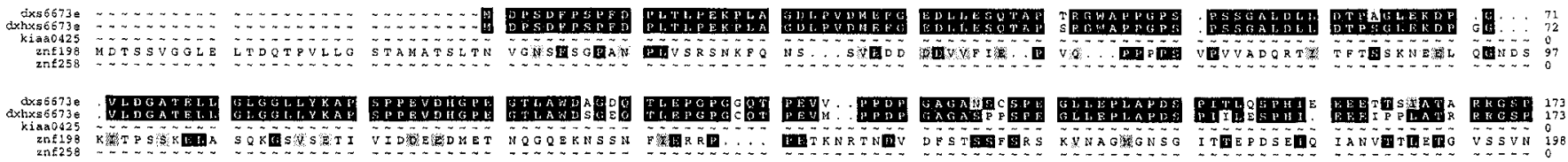

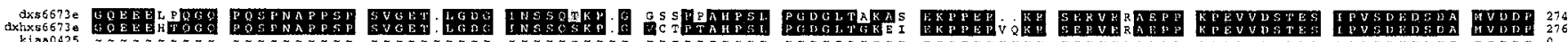

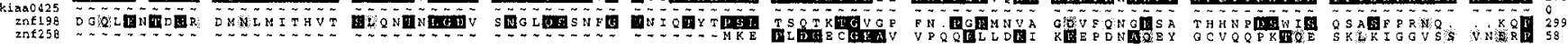

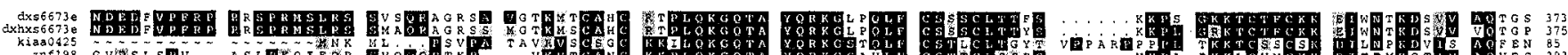

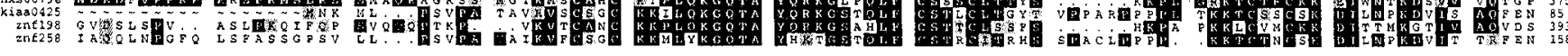

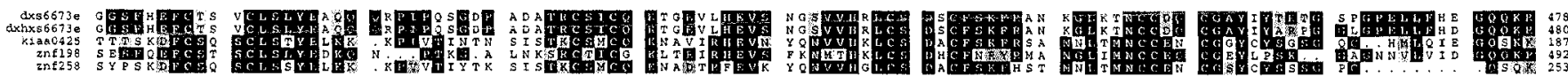

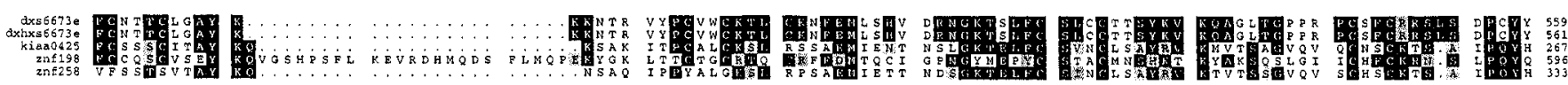

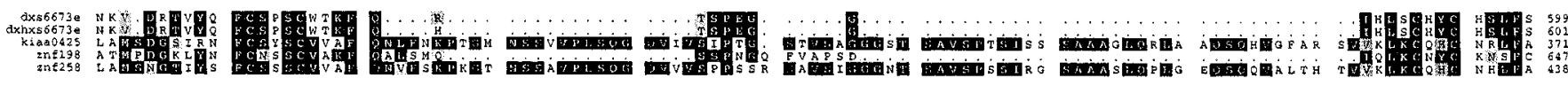

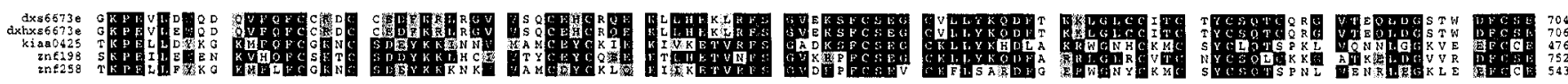

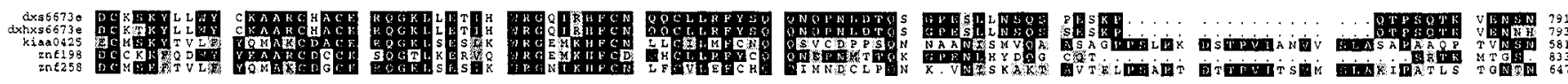

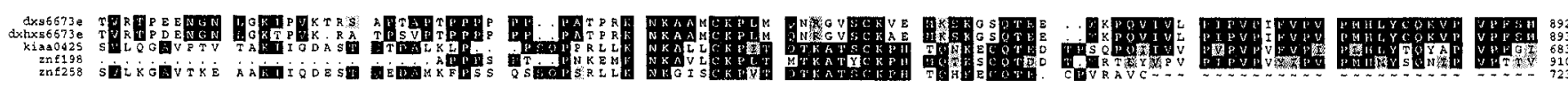

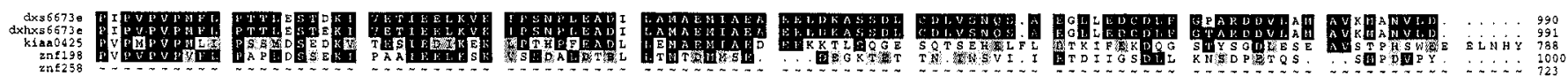

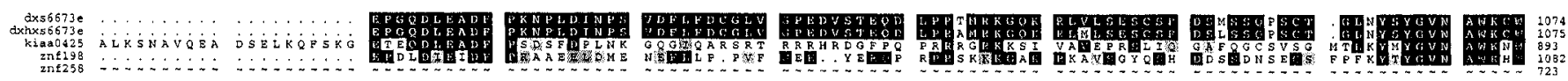

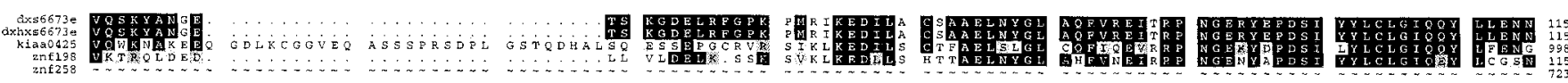

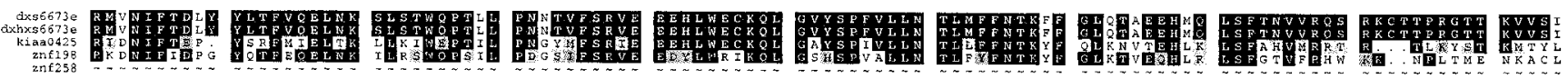

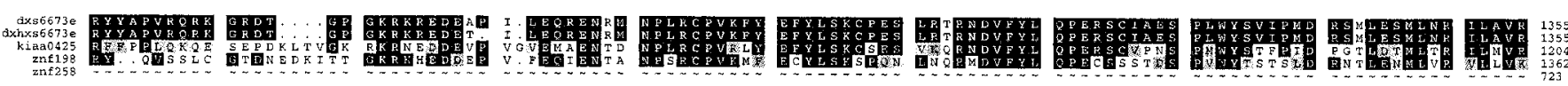

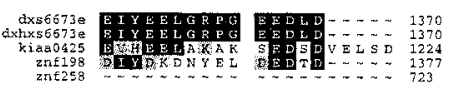

$\mathbf{B}$
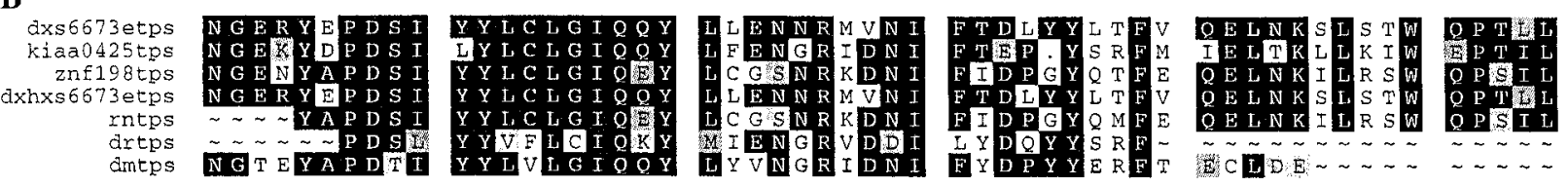

$1126-1180$ 974-1028 1133-1187 $1127-1181$ $2-51$ $36-68$ $80-125$

FIG. 1. Sequence alignment of members of the novel gene family. Amino acid sequences were compared using the Pileup program. Conserved amino acid residues are shaded. Numbers at the right indicate amino acid position (A) Significant sequence similarity over the entire length in mammals. DXS6673E (GenBank Accession No. X95808, a splice variant with 11 additional amino acids at position 797- 807, was used), DXHXS6673E (GenBank Accession No. AF 156605), KI AA0425 (GenBank Accession No. AB007885), ZNF 198 (GenBank Accession No. AJ 224901), and ZNF 258 (GenBank Accession No. AF 055470). The percentage identities between DXS6673E and other related proteins are 96\% (DXHXS6673E), 47\% (KIAA0425), 43\% (ZNF 198), and 37\% (ZNF258). (B) Evolutionary conservation of a putative tyrosine phosphorylation site. Rn, Rattus norvegicus (GenBank Accession No. Al 111670); Dr, Danio rerio (GenBank Accession No. Al397030); and Dm, Drosophila melanogaster (GenBank Accession No. AA567252). The percentage identities between the tyrosine phosphorylation site in DXS6673E and other related proteins are 100\% (DXHXS6673E), 65\% (KIAA0425), 68\% (ZNF 198), 65\% (Rntps), 52\% (Drtps), and 58\% (Dmtps).

ternative splice variants or correspond to the 1A variant, RT-PCR experiments were performed on RNA populations from various developmental stages $(12,14$,
16, and 18 days of gestation) and adult tissues (brain, kidney, liver, placenta, testis, and lung). To detect minor size variations, overlapping fragments between 0.9 


\section{Embryo}

$\mathrm{kb}$
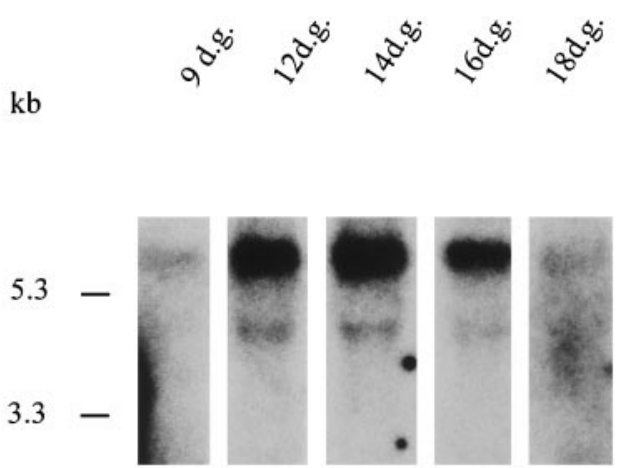

G3PDH
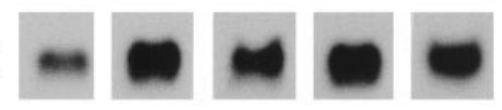

Adult

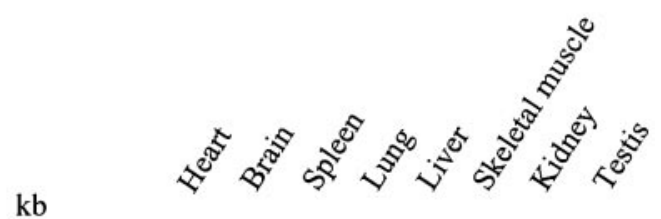

$\mathrm{kb}$
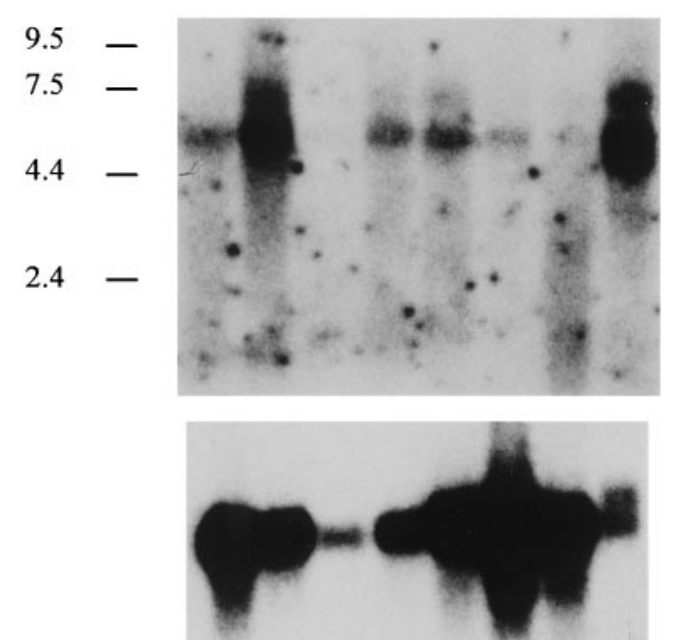

FIG. 2. Northern blot analysis. Hybridization of nucleotides 3385- 6173 of DXHXS6673E to a fetal (total RNA) and adult (poly(A) ${ }^{+}$RNA) Northern blot from several developmental stages or tissues. Age of embryo (days of gestation (d.g.) with day 1 as day of vaginal plug) and tissues are indicated. As a control, glyceraldehyde 3-phosphate dehydrogenase (G3PDH) cDNA was hybridized to the Northern blots.

and $1.4 \mathrm{~kb}$, starting from exon $1 \mathrm{~A}$ to exon 25 , were amplified. Products of unpredicted size were sequenced. Extensive alternative splicing was observed at the $5^{\prime}$-end of both $1 \mathrm{~A}$ and $1 \mathrm{~B}$ variants (Fig. 3). Exons 2 and 5 could be spliced out completely. In addition, exons 2 and 3 showed alternative internal donor splice site usage that removed 522 and 6 nt, respectively. The alternative splice site usage in exon 3 reconstitutes a NLS (aa 234-241) (Fig. 1). For 1B variants, the observed isoforms occurred, with the exception of $1 \mathrm{~B} \Delta 2 \Delta 3 \mathrm{i} \Delta 5$ and $1 \mathrm{~B} \Delta 2 \mathrm{i} \Delta 5$, in all stages of development and tissues analyzed (Fig. 3B). The dominant isoform corresponded to the full-length 1B transcript. I soforms lacking exon $5(\Delta 5)$, or 522 nt of exon $2(\Delta 2 \mathrm{i})$, are threeto fourfold less abundant, and exon 2 is completely absent in few transcripts. In contrast, 1A transcripts are more often alternatively spliced in a devel opmental stage and tissue-specific manner (Fig. 3A). In most RNA populations, the $5^{\prime}$ full-length 1 A variant represented the most prominent isoform. However, exon $1 \mathrm{~A}$ was not transcribed in liver, and in lung and placenta, only the 5' full-length $1 \mathrm{~A}$ variant or an isoform without exon 2 was present, respectively. I soforms $1 \mathrm{~A} \Delta 2 \mathrm{i}$ and $1 \mathrm{~A} \Delta 2 \mathrm{i} \Delta 3 \mathrm{i}$, derived from the internal donor splice site in exons 2 and 3, were detected only in embryos and in adult brain tissue, and splice variant $1 \mathrm{~A} \Delta 2 \Delta 5$ was detected in embryos at 14 days of gestation.

Between exons 6 and 25, only exon 23 was found to be alternatively spliced. This isoform was observed as a minor transcript in all RNA populations tested (data not shown).

Open reading frame (ORF) analysis of the isoforms revealed that they fell into three classes: (i) I n isoforms due to internal donor site usage in exon 2 and/or exon 3 , as represented by brain-specific transcripts $1 \mathrm{~A} \Delta 2 \mathrm{i}$ and $1 \mathrm{~A} \Delta 2 \mathrm{i} \Delta 3 \mathrm{i}$, the ORF is maintained. These variants lack amino acid residues 50 to 223 and/or 238 and 239. The ORF is also maintained in exon 23 isoforms. (ii) In splice variants without exon $5(\Delta 5)$, a shift in the ORF occurs and introduces a stop codon in exon 7 at position 86-88. These isoforms are translated into a shorter polypeptide containing an alternative carboxyl terminus of 87 novel amino acid residues. (iii) Splice variants without the entire exon 2 lack the original initiation codon AUG at position 717. The next start codon that fits the Kozak consensus sequence is situated in exon 7 in a different ORF and would give rise to a short polypeptide of 22 amino acid residues (MAAWYTDSAAILASPNSEPTRD). This peptide is identical to the C-terminus of $\Delta 5$ splice variants with the initiation codon in exon 2. However, the possibility is not excluded that $\Delta 2$ isoforms encode a truncated form of DXHXS6673E by a leaky ribosome scanning mechanism or by a non-AUG translational initiation as reported for the c-myc gene (Hann et al., 1988).

\section{Subcellular Localization of the DXS6673E Encoded Protein}

The presence of several putative nuclear targeting sequences in DXS6673E and its mouse ortholog suggested a nuclear localization of the proteins. To study their functional significance, fusion constructs with the GFP as reporter were analyzed. Using fluorescence 
A

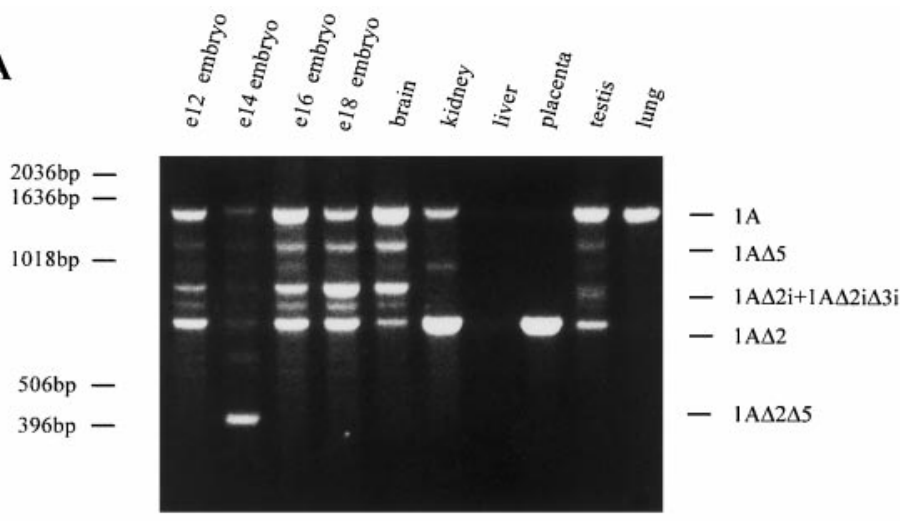

B

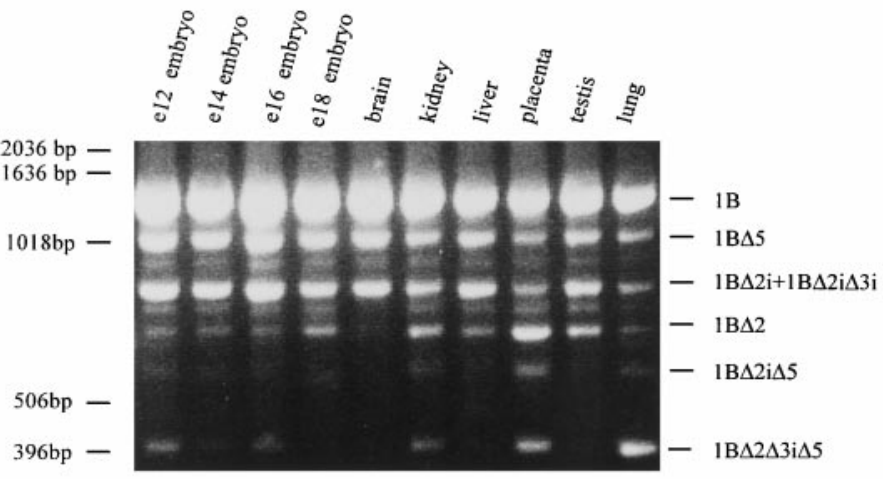

C

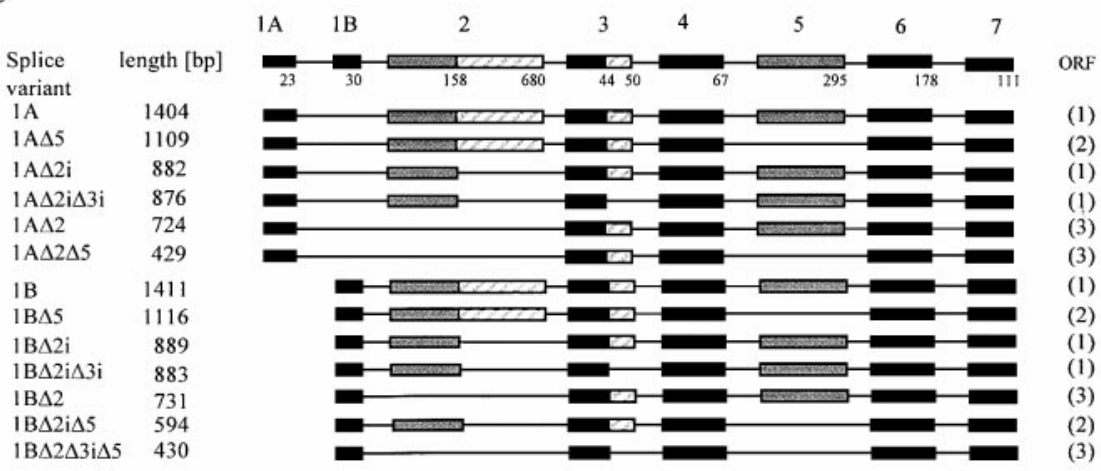

FIG. 3. Alternative splicing in mouse embryos and adult tissues. Nested RT-PCR using forward primers in exon $1 A$ and reverse primers in exon 7 (A) or exon 1B and exon 7 (B) was performed on various RNA populations. Cloned and sequenced products are indicated and correspond to splice variants listed in (C). Age of embryo (day of gestation) and adult tissues, used to isolate the respective RNA population, are shown above the lanes. (C) Schematic representation of splice variants at the 5'-end of DXHXS6673E. Names of splice variants and their sizes are given to the left. Constitutive exons are in black, alternatively spliced exons are in gray, and alternative donor splice sites are striped. Numbers above the exons indicate exon number; numbers below the exons indicate nucleotides within an exon. Exons $1 \mathrm{~A}, 1 \mathrm{~B}$, and 7 are not full-length due to internal PCR primers. (1) ORF maintained, (2) novel C-terminus of 87 amino acid residues, (3) alternative initiation codon.

microscopy, transient expression of fusion proteins in COS-7 cells revealed a dual localization in the cytoplasm and in the nucleus, with exclusion of the nucleol $i$ (Figs. 4A-4C). The distribution between these two compartments, however, varied within the cell population. In the majority of cells (47\%), fluorescence was strongest in the nucleus ( $\mathrm{F}$ igs. $4 \mathrm{~A}$ and 5 ). In one quarter, even distribution of the fluoresence was observed in the cell with a layer surrounding the nuclear enve- lope (Figs. 4B and 5), and in the remaining quarter, fluorescence was stronger in the cytoplasm, again mostly with a layer surrounding the nuclear envelope (Figs. 4C and 5). This distribution pattern did not vary over time $(24,48$, and $72 \mathrm{~h})$. Furthermore, in the DXS6673E constructs used (see also below), it was independent of the position of GFP. The only difference was a stronger fluorescence signal with $\mathrm{N}$-terminal GFP. 

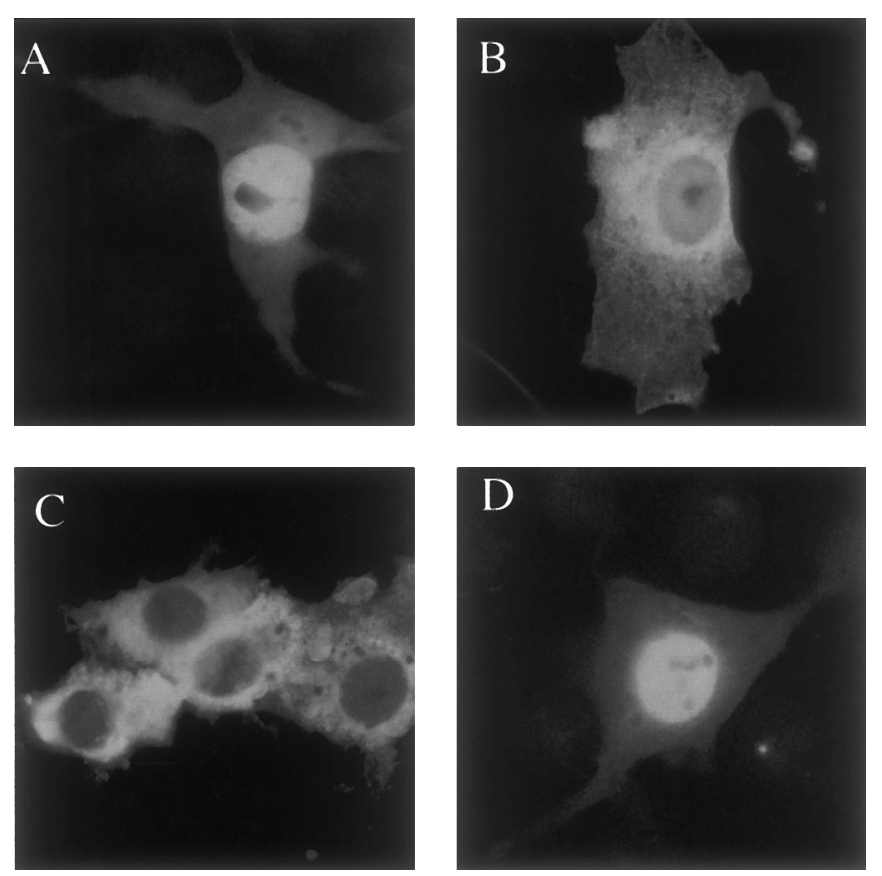

FIG. 4. Subcellular localization of GFP-DXS6673E fusion proteins in COS-7 cells. Different parts of the DXS6673E protein were fused to peGFP-C1 and analyzed $24 \mathrm{~h}$ after transfection into COS-7 cells for their subcellular localization. Cell type with predominant nuclear localization of the full-length fusion protein (A), cell type with even distribution of the full-length fusion protein (B), and cell type with predominant cytoplasmic localization and a strong perinuclear staining of the full-length fusion protein (C). Nuclear localization of truncated fusion protein $\Delta 474-1358$ (D). Subcellular localization was inspected $24 \mathrm{~h}$ after transfection in formaldehyde-fixed cells.

By comparison, GFP al one distributed in all cells analyzed throughout the nucleus and cytoplasm, since GFP $\left(M_{r}, 28,000\right)$ is able to diffuse passively into and out of the nucleus in mammalian cells (Ohno et al., 1998). As previously shown, staining was slightly stronger in the nucleus than in the cytoplasm (Lightfoot et al., 1999). This was also confirmed by subcellular fractionation and Western blot analysis (data not shown).
To quantify these results, subcellular fractionation was performed with subsequent Western blot analysis using an anti-GFP antibody. Most fusion protein was isolated with the nuclear fraction, whereas in the cytoplasmic supernatant, only a weak signal was obtained (Fig. 6). This result confirmed the predominant nuclear localization of the protein. It also supported the conceptual translation of DXS6673E into 1358 amino acid residues, since the observed molecular mass of the fusion protein is about 140-150 kDa higher than the molecular mass of the green fluorescent protein $\left(M_{r} 28,000\right)$ alone.

To analyze which of the NLSs are important for nuclear localization, we substituted the basic amino acid residues lysine and arginine in the first part of the bipartite NLS by two glycines, since they were shown to be important for nuclear targeting (Robbins et al., 1991). Fluorescence and subcellular fractionation studies in COS-7 cells, however, yielded no qualitative or quantitative differences in subcellular localization compared to wildtype (Fig. 5). To confirm that the bipartite NLS is indeed not necessary for nuclear transport, a truncated fusion protein was constructed containing the first 473 aa with two NLSs of the SV40 large $\mathrm{T}$ antigen type (construct $\Delta 474-1358$ ) (Fig. 5). This fusion protein was predominantly nuclear in all cells (Figs. 4D and 5). Hence, these NLSs are functional and can direct the transport of the DXS6673E protein into the nucleus. Interestingly, no cell types with a predominant cytoplasmic or even localization were observed (Fig. 5). To find out which of the amino acid residues are important for cytoplasmic localization, a further construct was analyzed containing the first 654 amino acids (construct $\Delta 655$ - 1358). Transfection yielded again three cell types with distinct protein distribution patterns. The ratio between these cell types, however, was altered (68:18:14) compared to the full-length protein (47:25:28) (Fig. 5). Nevertheless, this indicates that next to the NLSs, amino acid resi-

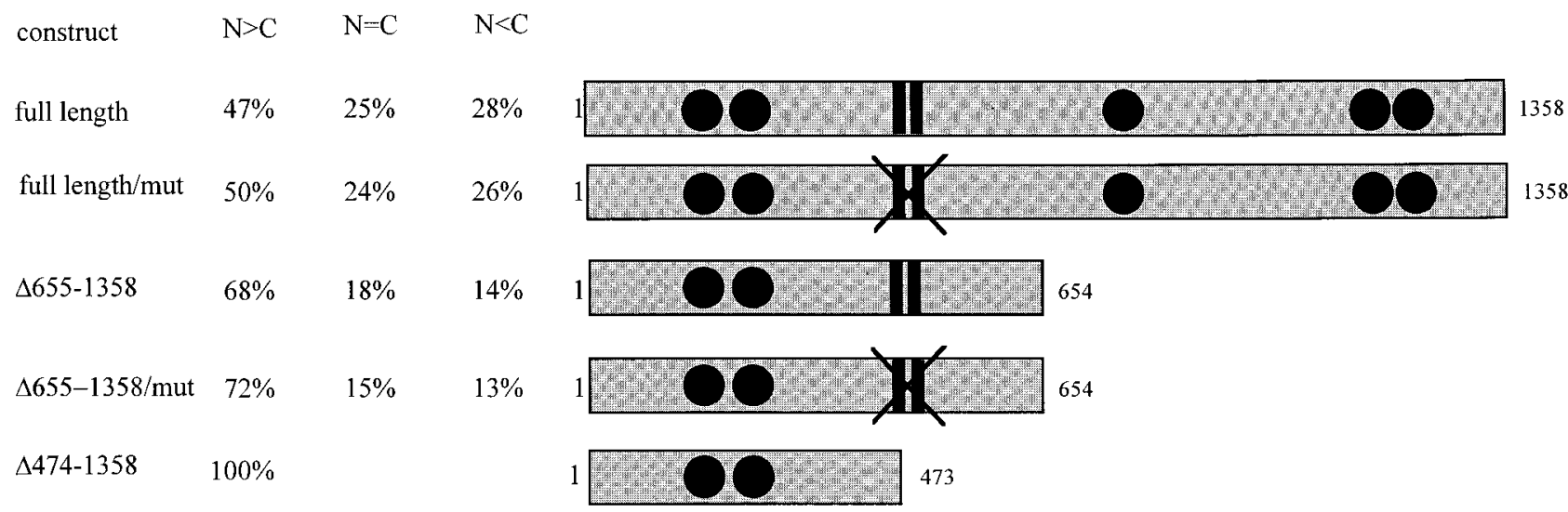

FIG. 5. Subcellular distribution types of DXS6673E fusion protein isoforms. Cell types were quantified by visually scoring $200-300$ clones/construct. $\mathrm{N}>\mathrm{C}$, predominantly nuclear localization; $\mathrm{N}=\mathrm{C}$, even staining in both cell compartments; $\mathrm{N}<\mathrm{C}$, predominantly cytoplasmic localization. Black circles represent the NLS of the SV40 large T antigen type, black bars represent the bipartite NLS, and crossed lines represent the mutated bipartite NLS. 


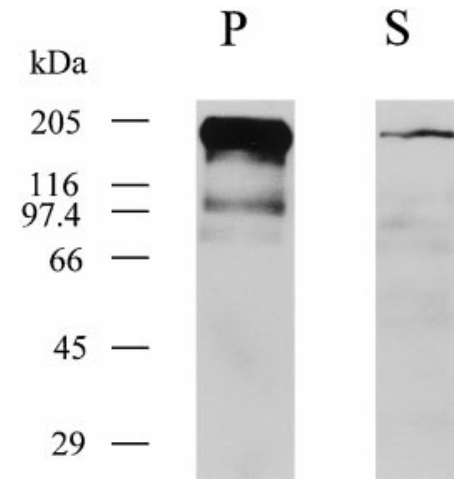

FIG. 6. Subcellular fractionation and immunoblot analysis. COS-7 cells were transfected with the pEGFPC1-DXS6673E fusion construct and harvested after $24 \mathrm{~h}$. Cells were lysed, and nuclei were separated from the cytosol by differential centrifugation. Equal volumes of both nuclear (P) and cytosolic (S) fractions were separated on a SDS-polyacrylamide gel, and proteins were electroblotted onto nitrocellulose. Blots were incubated first with anti-GFP antiserum and subsequently with horseradish peroxidase-conjugated anti-rabbit Fab fragments.

dues 474- 654 contain important information to guide subcellular distribution of the DXS6673E protein.

\section{DISCUSSION}

The mouse gene DXHXS6673E, isolated and characterized in this study, belongs to a novel, evolutionarily conserved gene family defined by a characteristic zincfinger motif. Two of the members, DXS6673E and ZNF 198, are likely involved in human diseases, but nothing is known about the function of the encoded proteins (van der Maarel et al., 1996; Xiao et al., 1998). In this regard, the cloning of the first mouse member will pave the way for functional analysis in an animal model, more amenable to genetic and molecular biology studies than human. Our identification of at least one family member in other model organisms like zebrafish and fruitfly will, in addition, help to assess basic gene functions. Surprisingly, no sequence similarity was detected in the almost completely sequenced genome of the nematode $\mathrm{C}$. elegans. Thus, this novel gene family may represent another family not present in nematodes, like genes of the hedgehog signaling pathway (Ruvkun and Hobert, 1998).

Expression studies showed the presence of two alternative 5'UTRs that give rise to $1 A$ and $1 B$ DSHXS6673E variants. Whereas exon 1B was more prominent and constitutively expressed in all developmental stages and tissues examined, transcription of exon 1A showed tissue specificity and occurred at a low level according to Northern blot analysis. These results, therefore, provide experimental proof for previous speculations based on a CpG island extending from human intron $1 \mathrm{~A}$ through exon $1 \mathrm{~B}$ into intron $1 \mathrm{~B}$ (van der Maarel et al., 1996). From the presence of this CpG island, a ubiquitous expression of variant $1 B$ and $a$ tissue-specific expression of variant 1A were hypothesized. Two alternative promoters might be, in general, characteristic for this gene family, since two independent transcription start sites were also reported for ZNF 198 (Reiter et al., 1998).

DSHXS6673E is also subject to highly alternative splicing at its $5^{\prime}$-end. The observed isoforms lead either to constitution of a NLS or to truncated proteins. Western blot analysis with DXS6673E-specific antibodies will show whether protein isoforms indeed exist or whether some of the characterized transcripts represent incorrectly spliced products. Several observations, however, argue against erroneously processed transcripts. (i) With the exception of exon 23, no further splice variants were observed in the other part of DXHXS6673E, but incorrect processing should a priori be random. (ii) RT-PCR of both total and poly(A) ${ }^{+}$RNA yiel ded the same proportion of splice variants (data not shown). (iii) Some of the splice variants were developmental stage or tissue specific. For example, alternative use of the exon 2 internal donor splice site in $1 \mathrm{~A}$ transcripts occurred only in adult brain tissue, whereas the same splice site was alternatively used in 1B variants of all tissues examined. (iv) The ratio of alternative splicing of a given exon varied between $1 \mathrm{~A}$ and $1 \mathrm{~B}$ transcripts. I soform $\Delta 2$ was a minor $1 \mathrm{~B}$, but a prominent IA variant. (v) A splice variant of ZNF 198 was also described that disrupts the ORF between the $\mathrm{N}$-terminus and the fourth $\mathrm{Zn}$-finger motif by introducing a stop codon before Met 621 (Reiter et al., 1998). Furthermore, exon 2 and exon 3 of ZNF 198 are variably incorporated into the mature transcript, and exon 4 contains an internal splice site (Kulkarni et al., 1999). Thus, highly alternative splicing might be a general feature of this novel gene family.

Interestingly, two prominent splice variant classes are complementary: they either lack $(\Delta 2 \mathrm{i})$ or translate only into the $\mathrm{N}$-terminus $(\Delta 5)$. If DXHXS6673E functions as a transcription factor, this alternative processing might modulate its activity as reported for some proteins. A C-terminal truncated form of F osB inhibits Fos/J un transcriptional activity, and a liver-enriched protein acts as either a transcriptional activator (LAP) or an inhibitor (LIP), depending on its alternative Nterminus (Nakabeppu and Nathans, 1991; Descombes and Schibler, 1991). LIP attenuates the transcriptional stimulation by LAP at substoichiometric amounts and is likely involved in fine-tuning of transcription factor activity (Descombes and Schibler, 1991). The alternatively spliced N-terminus of DXHXS6673E contains one putative NLS and several short proline-rich sequences, one of which occurs twice in the orientation PPSP and once in the opposite orientation, PSPP. F urthermore, the internal splicing in exon 2 disrupts another proline-rich sequence, WAPPGPSP, similar to a peptide previously shown to bind the $\mathrm{SH} 3$ domain (Pawson, 1995). It will, therefore, be interesting to see whether the $\mathrm{N}$-terminus is involved in protein-protein interactions and is important for the function of DXHXS6673E.

A splice variant without the initiation codon in exon 
$2(1 \mathrm{~A} \Delta 2 \Delta 5)$ represented the dominant $1 \mathrm{~A}$ transcript in the mouse embryo at 14 days of gestation. At this stage, comparable to a 6-week-old human embryo, most of the major organs have been laid down, and future development is largely a matter of refinement and cellular differentiation. The nervous system is composed largely of differentiating neuroblasts. A detailed study of the diencephalon showed that the second stage of nuclear development in the diencephalic wall occurs from 13 to 15 days of gestation with differentiation of the various layers: germinal, mantle, and marginal (Rugh, 1990). Two other isoforms, $1 A \Delta 2 i$ and $1 \mathrm{~A} \Delta 2 \mathrm{i} \Delta 3 \mathrm{i}$, were observed only during development and in adult brain. This tissue-specific transcription and alternative splicing of variant $1 \mathrm{~A}$ might be important with respect to the human DXS6673E. In this gene, disruption of exon $1 \mathrm{~A}$ by a balanced translocation is associated with NSXMR, even though sequences $3^{\prime}$ of the breakpoint, and thus the ORF, are still expressed. Lack of the X-chromosomal promoter and part of exon IA might lead to altered expression pattern, splicing, stability, or transport of $1 \mathrm{~A}$ variants and hence influence brain development and functioning.

Since it is not known whether the $\mathrm{Zn}$-finger motifs found in this novel family mediate DNA-protein or protein-protein interactions, it was important to study the subcellular localization of one of its members. The analysis of GFP-DXS6673E fusion proteins demonstrated a predominant nuclear localization. Due to the molecular weight of the various fusion proteins examined $\left(M_{r}>75,000\right)$, an active transport must occur, induced by nuclear targeting sequences (Ohno et al., 1998). Our analysis of DXS6673E revealed five NLSs of the SV40 type and one bipartite NLS that may direct the nuclear transport. The most reliable indicator of nuclear localization, the bipartite motif, is present in more than $50 \%$ of nuclear proteins, while it is present in less than $5 \%$ of nonnuclear proteins (Dingwall and Laskey, 1991). The fact that its mutation did not alter the subcellular distribution of the fusion protein is likely due to the presence of additional functional nuclear targeting signals. That the bipartite NLS is dispensable for nuclear localization is supported by sequence analysis, since it is not present in other family members (ZNF198 and ZNF 258). This signal might, therefore, be involved in fine-tuning of the nuclear transport of DXS6673E and DXSHXS6673E proteins.

Three different subtypes of cells could be identified according to the nuclear-cytoplasmic distribution of the fusion protein. Whether this observation reflects any physiological function like cell cycle dependency, and whether the elevated cytoplasmic concentration in some cells represents rather a retention of the synthesized protein in the cytoplasm or a nuclear export of the protein, is the object of current studies.

In summary, the data presented here show that DXS6673E and its mouse ortholog DXHXS6673E are members of an evolutionarily conserved family of zincfinger proteins. The cloning of DXHXS6673E will facil- itate genetic and functional analysis in an animal model to elucidate the precise role of this novel family further.

\section{ACKNO WLEDGMENTS}

Wethank S. Schneider and D. Vogt for expert technical assistance. $H$. Madle and S. Freier are gratefully acknowledged for hel p with the cell culture. We are grateful to M. Hemberger, R. Kirschner, and S. Lenzner for providing mouse embryos and RNA from various mouse tissues and to the German Ressource Center for providing genomic $\mathrm{P} 1$ clones. This work was supported by the Bundesministerium für Bildung und Forschung (Grant 4763 to H. H. Ropers).

\section{REFERENCES}

Allen, K. M., Gleeson, J. G., Bagrodia, S., Partington, M. W., MacMillan, J . C., Cerione, R. A., Mulley, J . C., and Walsh, C. A. (1998). PAK3 mutation in nonsyndromic X-linked mental retardation. Nat. Genet. 20: 25-30.

Billuart. P., Bienvenu. T., Ronce. N., des Portes, V., Vinet, M. C., Zemmi, R., Crollius, H. R., Carrie, A., Fauchereau, F., Cherry, M., Briault, S., Hamel, B., Fryns, J. P., Beldjord, C., Kahn, A., Moraine, C., and Chelly, J . (1998). Oligophrenin-1 encodes a rhoGAP protein involved in X-linked mental retardation. Nature 392: 823826.

Chomczynski, P., and Sacchi, N. (1987). Single-step method of RNA isolation by acid guanidinium thiocyanate-phenol-chloroform extraction. Anal. Biochem. 162: 156-159.

d'Adamo, P., Menegon, A., Lo J igro, C., Grasso, M., Gulisano, M., Tamanini, F., Bienvenu, T., Gedeon, A. K., Oostra, B., Wu, S. K., Tandon, A., Valtorta, F., Balch, W. E., Chelly, J ., and Toniolo, D. (1998). Mutations in GDI 1 are responsible for X-linked non-specific mental retardation. Nat. Genet. 19: 134-139.

Descombes, P., and Schibler, U. (1991). A liver-enriched transcriptional activator protein, LAP, and a transcriptional inhibitory protein, LIP, are translated from the same mRNA. Cell 67: 569579.

Devereux, J ., Haeberli, P., and Smithies, O. (1984). A comprehensive set of sequence analysis programs for the VAX. Nucleic Acids Res. 12: 387-395.

Dingwall, C., and Laskey, R. A. (1991). Nuclear targeting sequences-A consensus? Trends Biochem. Sci. 16: 478- 481.

Dingwall, C., and Laskey, R. A. (1998). Nuclear import: A tale of two sites. Curr. Biol. 8: 922-924.

Gecz, J ., Gedeon, A. K., Sutherland, G. R., and Mulley, J . C. (1996). I dentification of the gene FMR2, associated with FRAXE mental retardation. Nat. Genet. 13: 105-108.

Hann, S. R., King, M. W., Bentley D. L., Anderson C. W., and Eisenman, R. N. (1988). A non-AUG translational initiation in c-myc exon-1 generates an $\mathrm{N}$-terminally distinct protein whose synthesis is disrupted in Burkitt's lymphomas. Cell 52: 185-195.

Kulkarni, S., Reiter, A., Smedley, D., Goldmanm J . M., and Cross, N. C. (1999). The genomic structure of ZNF 198 and location of breakpoints in the $t(8 ; 13)$ myeloproliferative syndrome. Genomics 55: $118-121$.

Lightfoot, J ., Alon, N., Bosnoyan-Collins, L., and Buchwald, M. (1999). Characterization of regions functional in the nuclear localization of the Fanconi anemia group A protein. Hum. Mol. Genet. 8: $1007-1015$.

Nakabeppu, Y., and Nathans, D. (1991). A naturally occurring truncated form of FosB that inhibits Fos/] un transcriptional activity. Cell 64: 751-759.

Ohno, M., Fornerod, M., and Mattaj, I. W. (1998). Nucleocytoplasmic transport: The last 200 nanometers. Cell 92: 327-336. 
Pawson, T. (1995). Protein modules and signalling networks. Nature 373: 573-580.

Popovici, C., Adelaide, J., Ollendorff, V., Chaffanet, M., Guasch, G., J acrot, M., Leroux, D., Birnbaum, D., and Pebusque, M. J . (1998). Fibroblast growth factor receptor 1 is fused to FIM in stem-cell myeloproliferative disorder with $\mathrm{t}(8 ; 13)$. Proc. Natl. Acad. Sci. USA 95: 5712-5717.

Reiter, A., Sohal, J ., Kulkarni, S., Chase, A., Macdonald, D. H., Aguiar, R. C., Goncalves, C., Hernandez, J. M., J ennings, B. A., Goldman, J. M., and Cross, N. C. (1998). Consistent fusion of ZNF 198 to the fibroblast growth factor receptor-1 in the t(8; 13)(p11;q12) myeloproliferative syndrome. Blood 92: 17351742.

Robbins, J ., Dilworth, S. M., Laskey, R. A., and Dingwall, C. (1991). Two interdependent basic domains in nucleoplasmin nuclear targeting sequence: Identification of a class of bipartite nuclear targeting sequence. Cell 64: 615-623.

Rugh, R. (1990). "The Mouse: Its Reproduction and Development," Oxford Univ. Press, Oxford.
Ruvkun, G., and Hobert, O. (1998). The taxonomy of developmental control in Caenorhabditis elegans. Science 282: 2033-2040.

Sambrook, J ., Fritsch, E. F., and Maniatis, T. (1989). “Molecular Cloning: A Laboratory Manual, Cold Spring Harbor Laboratory Press, Cold Spring Harbor, NY.

Smedley, D., Hamoudi, R., Clark, J., Warren, W., Abdul-Rauf, M., Somers, G., Venter, D., Fagan, K., Cooper, C., and Shiple, J . (1998). The $t(8 ; 13)(p 11 ; q 11-12)$ rearrangement associated with an atypical myeloprol iferative disorder fuses the fibroblast growth factor receptor 1 gene to a novel gene RAMP. Hum. Mol. Genet. 7: 637- 642.

van der Maarel, S. M., Scholten, I. H., Huber, I., Philippe, C., Suijkerbuijk, R. F., Gilgenkrantz, S., Kere, J ., Cremers, F. P., and Ropers, H. H. (1996). Cloning and characterization of DXS6673E, a candidate gene for X-linked mental retardation in Xq13.1. Hum. Mol. Genet. 5: 887- 897.

Xiao, S., Nalabolu, S. R., Aster, J . C., Ma, J ., Abruzzo, L., J affe, E. S., Stone, R., Weissman, S. M., Hudson, T. J ., and Fletcher, J . A. (1998). FGFR1 is fused with a novel zinc-finger gene, ZNF 198, in the $(8 ; 13)$ leukemia/lymphoma syndrome. Nat. Genet. 18: 84-87. 